\title{
TG17
}

\section{Data Collection Strategy for Exploration Wells in Tight Reservoirs}

\section{E. Beretta* (Eni E\&P)}

\section{SUMMARY}

Tight and ultra tight gas reservoirs are recognised as providing significant opportunities for oil companies worldwide to add producible reserves to their portfolios. However, it is commonly perceived that wells in such reservoirs may suffer from low productivity and possibly unstable rates below the economic threshold.

Massive hydraulic fracturing treatments are considered to be the most effective operations to improve well productivities and achieve economic flow rates in these environments. These operations can sometimes be complex and expensive actions where costs can sometimes be in the order of millions of US\$. Exploiting tight reservoirs through such stimulation treatments may thus only be attractive in a promising hydrocarbon price scenario.

Inarguably, conventionally acquired well data such as cores, logs, wireline and testing etc. do assist in driving decisions whether or not to fracture, but usually fall short of providing reliable information about the most likely dynamic reservoir and fluid response.

In this respect, especially during the exploration and appraisal phases, a clear strategy has to be planned a priori to determine the most suitable approach that maximises the value of information about the reservoir dynamic behaviour and indicates whether an expensive fracturing treatment may be beneficial or not.

A general workflow will be presented with the aim of facilitating the decision making process. 